\title{
Fluctuation-Induced Excess Conductivity and Infrared Spectra in Y Doped BSCCO Superconductors
}

\author{
A. Sedky ${ }^{*}$, A. T. AlMotasem \\ Physics department, Faculty of Science, Assiut University,71516 Assiut, Egypt \\ Email: sedky196000@hotmail.com; sedky1960@yahoo.com
}

Received: 18 March 2019; Accepted: 20 April 2019; Available online: 13 May 2019

\begin{abstract}
We report here the fluctuation induced excess conductivity and IR spectra in $\mathrm{Bi}_{2} \mathrm{Sr}_{2} \mathrm{Ca}_{1-\mathrm{x}} \mathrm{Y}_{\mathrm{x}} \mathrm{Cu}_{2} \mathrm{Oy}(0.00$ $\leq \mathrm{X} \leq 0.50$ ) superconductors. This work is done by using the reported data of Sedky, Physica B 410, 227 (2013), and with the help of Anderson and Zou relation. The logarithmic plots of excess conductivity $\Delta \sigma$ and reduced temperature $\epsilon$ reveal two different exponents corresponding to unique crossover temperature in the slope of each plot. The first exponent is obtained in the normal field region at a temperature of $\left(T_{c}^{\mathrm{mf}}<<\mathrm{T}<2 T_{c}^{\mathrm{mf}}\right)$, while the second exponent is obtained in the mean field region at a temperature of $\left(\mathrm{T} \sim T_{c}^{\mathrm{mf}}\right)$. The dimensional exponents are shifted from three dimensional (3D) to two dimensional (2D) for $\mathrm{x} \leq 0.30$, but it is shifted from 2D to 3D for $\mathrm{x}=0.50$. Both zero kelvin critical magnetic fields and current density are considerably enhanced by increasing $\mathrm{x}$ up to 0.30 followed by a decrease with further increase of $x$ up to 0.50 . The vice is versa for the behavior of interlayer coupling, coherence lengths and anisotropy against $\mathrm{x}$. On the other hand, IR spectra show absorption modes in the wave number range of $716-726 \mathrm{~cm}^{-1}$ according to the value of $\mathrm{x}$. These results are discussed in terms of the correlation between carrier concentration, oxygen deficient and effective $\mathrm{Cu}$ valance which are induced by $\mathrm{Y}$ through $\mathrm{CuO}_{2}$ planes of BSCCO superconductors.
\end{abstract}

Keywords: $Y$ substitution; Conductivity; BSCCO; Critical temperature; Infrared.

\section{Introduction}

The high temperature superconductors have a layered structure in which two dimensional conducting $\mathrm{CuO}_{2}$ planes are separated by $\mathrm{Bi}$ charge reservoir layers, which impede the movement of carriers normal to the conducting planes [1-7]. In particular, these types of materials exhibit anisotropy and small coherence length together with elevated values of critical temperature Tc. These materials have a significant effect on the fluctuations of superconducting order parameter, which have been early observed in the conductivity versus temperature measurements as excess conductivity. However, the fluctuation induced excess conductivity is an important parameter experimentally accessible method for exploring the normal state properties above Tc [3, 8$10]$.

The fluctuation induced conductivity (FIC) analyses reveal that the contribution of excess conductivity is due to Gaussian fluctuation in the mean field region as well as the critical fluctuation region [11]. Gaussian fluctuation is probably dominant in the temperature region above the mean field temperature $T_{c}^{\mathrm{mf}}$ when the fluctuation in the order parameter is small and the interactions between Cooper pairs can be neglected. While the critical fluctuation occurs in the critical field region below the $T_{c}^{\mathrm{mf}}$ when the fluctuation in the order parameter is large and the interactions between Cooper pairs is considered. The variation of excess conductivity with the reduced temperature helps the researchers to find the crossover temperatures, dimensional exponents, interlayer coupling, coherence lengths and anisotropy [12]. The dimensional exponents in high $\mathrm{T}_{\mathrm{C}}$ materials are found to be zero dimensional (0D), one dimensional (1D), two dimensional (2D) and three dimensional (3D) [13, 14]. It seems that the dimensional crossover takes place between any two different dimensions and is mainly obtained above $\mathrm{T}_{C}$ at a crossover temperature $T_{0}$. It is found that the superconducting order parameter of BSCCO samples are 2D dimensional [15-19], and the crossover is occurred either from 3D to 2D or from 1D to 2D in the doped samples [20].

The lattice vibrations in ceramic cuprates have been considered early as the subject of numerous studies and applications such as optical Kerr shutter (OKS), switching broad-band amplifiers, detectors and many other switching devices. Now, some evidences for electron- phonon coupling have been reported by infrared spectroscopy. Most of IR-active phonon studies based on high Tc superconductors are focused on the frequency region of 50-520 cm-1, and few reports in frequency region of $600-700 \mathrm{~cm}-1$ [21-23]. Bi: 2212 high Tc system is 
potentially one of the most appropriate systems for the study of impurity doping at the Ca site. In order to distinguish the different roles between the spin vacancy and the carrier concentration, the effect of impurity doping on the infrared spectra is considered. However, IR spectra of the samples in powder form are carried out by using IR unit in which $\mathrm{KBr}$ is used as a carrier. The powdered samples are homogenized in spectroscopic grade $\mathrm{KBr}$ in an agate mortar and pressed in to $3 \mathrm{~mm}$ pellets with a hand press. We tried to minimize the grinding time to avoid as possible the deformation of the crystals structure, the ion exchange and the water absorption from atmosphere. Infrared spectra have been measured at room temperature for the multiphase superconducting samples, and the IR spectra display structures at $\sim 590 \mathrm{~cm}-1$ and $\sim 530 \mathrm{~cm}-1$. The difference of IR spectra can be taken as an indicator for higher Tc superconductor when the difference between them is small.

Recently Sedky et. al. [24] have investigated the effect of Y substitution on the properties of $\mathrm{Bi}_{2} \mathrm{Sr}_{2} \mathrm{Ca}_{1}$ ${ }_{\mathrm{x}} \mathrm{Y}_{\mathrm{x}} \mathrm{Cu}_{2} \mathrm{Oy}$ superconductors. The results of $\mathrm{XRD}$, resistivity, effective $\mathrm{Cu}$ valence and hole carriers/ Cu ions have been presented in details. It is found that the critical temperature, hardness and surface energy are improved by $\mathrm{Y}$ addition up to 0.30 , followed by a decrease with further increase of Y up to 0.50. As a continuation of the above work, we reported here the fluctuation induced conductivity produced by $\mathrm{Y}$ substitution on the same batch of samples. We have restricted our analysis to the mean field regime and crossover behavior and tried to extract some physical parameters such as coherence lengths, anisotropy, interlayer coupling, dimensional exponent, critical magnetic field and critical current density. Furthermore, The IR spectra are taken in the frequency range from 40$800 \mathrm{~cm}^{-1}$ and the results are compared with the excess conductivity analysis.

\section{Theoretical background}

The excess conductivity $\Delta \sigma$ due to thermal fluctuation is defined as the deviation of the measured conductivity of $\sigma_{\mathrm{m}}(\mathrm{T})$ from the normal conductivity $\sigma_{\mathrm{n}}(\mathrm{T})$ as follows:

$$
\Delta \sigma=\left(\frac{1}{\rho_{m}}-\frac{1}{\rho_{n}}\right)=\sigma_{m}-\sigma_{n}
$$

where $\rho_{\mathrm{m}}$ and $\rho_{\mathrm{n}}$ are the measured and normal resistivity, respectively. $\rho_{\mathrm{n}}$ is obtained from the measured resistivity $\rho_{\mathrm{m}}$ at $\mathrm{T} \geq 2 \mathrm{~T}_{\mathrm{c}}$ by applying the least square method to the Anderson and Zou relation, $\rho_{n}(T)=A+B T$ [26]. In order to estimate the paraconductivity, Aslamazov and Larkin (AL) deduced the following relation for the fluctuation induced excess conductivity $\Delta \sigma[25]$ as:

$$
\Delta \sigma=A \epsilon^{-\lambda}
$$

here, $A=e^{2} / 32 \hbar \xi_{c}(0)$ for $3 \mathrm{D}, A=e^{2} / 16 \hbar d$ for $2 \mathrm{D}, A=e^{2} \xi_{c}(0) / 32 \hbar s$ for $1 \mathrm{D}$, e is the electronic charge, $\mathrm{d}$ is the interlayer spacing between two successive $\mathrm{CuO}_{2}$ planes, $\hbar$ is the reduced Planks' constant, $\xi_{\mathrm{c}}(0)$ is the c-axis $3 \mathrm{D}$ coherence length at zero temperature, $\mathrm{s}$ is the wire cross-sectional area of the 1D system, $\lambda$ is an exponent of dimensionality, and their values are $0.5,1$ and 1.5 and $\geq 2$ for 3D, 2D, $1 \mathrm{D}$ and $0 \mathrm{D}$ fluctuations respectively, and $\epsilon$ is the reduced temperature given by [25-27]:

$$
\varepsilon=\frac{T-T_{c}^{m f}}{T_{c}^{m f}}
$$

where $T_{c}^{\mathrm{mf}}$ is the mean field temperature, above it the interactions between Cooper pairs can be neglected. We have followed the $\mathrm{d} \rho / \mathrm{dT}$ versus $\mathrm{T}$ plot to obtain the values of $T_{c}^{\mathrm{mf}}$ from the peaks.

For polycrystalline samples, the modified equations for 2D and 3D fluctuations are expressed as [28]:

$$
\begin{aligned}
& \Delta \sigma_{3 D}=\frac{e^{2}}{32 \hbar \xi_{p}(0)} \varepsilon^{-\frac{1}{2}} \\
& \Delta \sigma_{2 D}=\frac{1}{4}\left\{\frac{e^{2}}{16 \hbar d} \varepsilon^{-1}\left[1+\left(1+\frac{8 \xi_{c}^{4}(0)}{d^{2} \xi_{a b}^{2}(0)} \varepsilon^{-1}\right)^{\frac{1}{2}}\right]\right\}
\end{aligned}
$$

where $\xi_{\mathrm{ab}}(0)$ is the coherence length at $0 \mathrm{~K}$ across the ab- plane and $\xi_{\mathrm{p}}(0)$ is the effective characteristic coherence length at $0 \mathrm{~K}$. On the other hand, the cross over behavior from 2D-3D occurs at a temperature $\mathrm{T}_{0}$ given by [27]:

$$
T_{0}=T_{c}^{m f} \exp \left(\frac{2 \xi_{c}(0)}{d}\right)^{2}
$$

where $\xi_{c}(0)$ is given by [29, 30]: 


$$
\xi_{c}(0)=\left(\frac{d k^{\frac{1}{2}}}{2}\right)
$$

where $k$ is the interlayer coupling, and expressed by [31, 32]:

$$
k=\ln \left(\frac{T_{0}}{2 T_{c}^{m f}}\right)
$$

\section{Results and discussion}

The resistivity versus temperature curves of the considered samples are given in Figure 1 (a - e). The normal resistivity is found to be linear as the temperature is reduced from room temperature down to a certain temperature $\mathrm{T}_{\mathrm{B}}$. In this region $\rho_{n}(T)$ follows the above formula, $\rho_{n}(T)=A+B T$ as discussed above. $\mathrm{T}_{\mathrm{B}} \sim 2 T_{c}^{\mathrm{mf}}$ is defined as the temperature below which the Cooper-pair formation starts [10, 33]. As the temperature is further reduced beyond the normal state region, the rate of change of resistivity becomes entirely different as compared to this region. This is mainly due to increasing Cooper pair formation as the temperature is reduced. Therefore, the fluctuation induced conductivity in this region follows the Aslamazov and Larkin (A-L model) [27] to yield the dimensional exponent appropriate to fluctuation-induced conductivity in these samples.

However, $\rho_{n}(T)$ is calculated by using the values of A and B parameters, which are obtained from the fitting as listed in Figure 1. We have extrapolated the linear fit of the normal state resistivity to the lower temperature regime as shown by straight lines in Figure 1. The straight columns drawn in the curves indicate the width of temperatures fitting. One of them occurs at a temperature close to $T_{B}$ and the second at a temperature very close to room temperature. The mean field temperatures $T_{c}^{\mathrm{mf}}$ for all samples are estimated from the peak of $\mathrm{d} \rho / \mathrm{dT}$ against temperature plot, see Figure 2. Similar values are listed in Table 1. By using the values of $\Delta \sigma$ and reduced temperatures $\mathrm{C}$, we have plotted $\ln \Delta \sigma$ against $\ln \mathrm{C}$ for all samples, see Fig. 3. It is evident from the fitting that there is one distinct change in the slope of each plot close to the mean field region. The corresponding temperature where the slope change occurs is designated as the crossover temperature $T_{0}$. Therefore, the crossover temperatures along with two different exponents are obtained from each plot with an accuracy of $\pm 1 \mathrm{~K}$. Anyhow, the different values of $T_{c}, T_{c}^{\mathrm{mf}}$ and $\mathrm{T}_{\mathrm{o}}$ against $\mathrm{Y}$ content are shown in Figure 4 (a). Similar values are listed in Table 1 . It is evident from the Figure that both $\mathrm{T}_{\mathrm{c}}, T_{c}^{\mathrm{mf}}$ and $\mathrm{T}_{\mathrm{o}}$ are increased by increasing $\mathrm{x}$ up to 0.30 , followed by a decrease with further increase of $\mathrm{x}$ up to 0.50 .
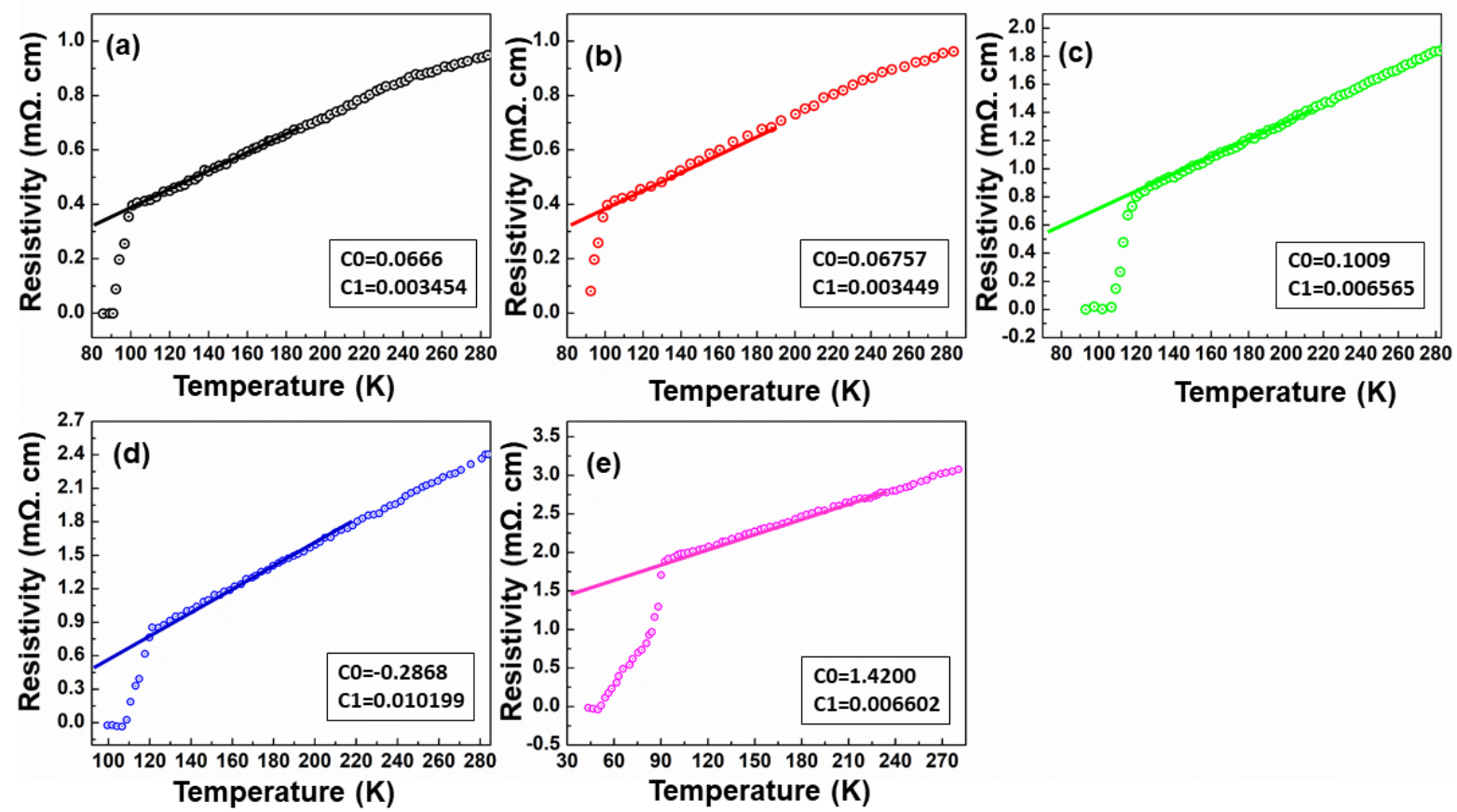

Figure 1. Resistivity versus temperature for (a) pure, (b) $x=0.075$, (c) $x=0.15$, (d) $x=0.3$ and (e) $x=0.5$ samples

The first exponent is obtained in the normal region above mean field region at a temperature range of $(-1.10 \geq$ $\ln \epsilon \geq-2.04)$ for $x=0.00,(-1.05 \geq \ln \epsilon \geq-2.30)$ for $x=0.075,(-1.30 \geq \ln \epsilon \geq-1.98)$ for $x=0.15,(-1.02 \geq \ln \epsilon$ $\geq-2.31)$ for $x=0.30,(-0.79 \geq \ln C \geq-1.53)$ for $x=0.50$. The exponent values are $0.57(3 \mathrm{D}), 0.39$ (3D), 0.44 (3D), 
0.46 (3D) and 1.10 (2D) for all samples, respectively. This indicates that the OD is 3D $\mathrm{x}=0.00,0.075,0.15$ and 0.3 and $2 \mathrm{D}$ for $\mathrm{x}=0.50$ sample. While, the second exponent is obtained in the mean field region above $\mathrm{T}_{\mathrm{C}}$ at a temperature range of $(-2.22 \geq \ln \epsilon \geq-3.83)$ for $x=0.00,(-2.59 \geq \ln C \geq-3.68)$ for $x=0.075,(-0.2 .15 \geq \ln C \geq$ - 4.03) for $\mathrm{x}=0.15,(-2.31 \geq \ln \epsilon \geq-4.06)$ for $\mathrm{x}=0.30,(-2.22 \geq \ln \mathrm{C} \geq-3.61)$ for $\mathrm{x}=0.50$. The exponent values are 1.08 (2D), 0.96 (2D), 1.05 (2D), 1.02 (2D) and 0.58 (3D) for all samples, respectively. This indicates that the $0 \mathrm{D}$ is quasi- 2D for $\mathrm{x}=0.50$ sample, and 2D for the rest of samples. However, Figure 4(b) shows the variation of the order parameter as a function of Y content, and similar values are listed in Table 1 . These results indicate that the crossover occurred from 3D to 2D for all samples except $\mathrm{x}=0.50$ sample, in which the crossover occurred from $2 \mathrm{D}$ to quasi- $2 \mathrm{D}$. This is in good agreement with the behaviors of $\mathrm{T}_{\mathrm{c}}, T_{c}^{\mathrm{mf}}$ and $\mathrm{T}_{\mathrm{o}}$ for this sample, and also consistent with the reported data for true hardness and surface energy [24]. To our knowledge the analysis of fluctuation induced conductivity for the Y substituted at Ca site in Bi:2212 may be reported for the first time.

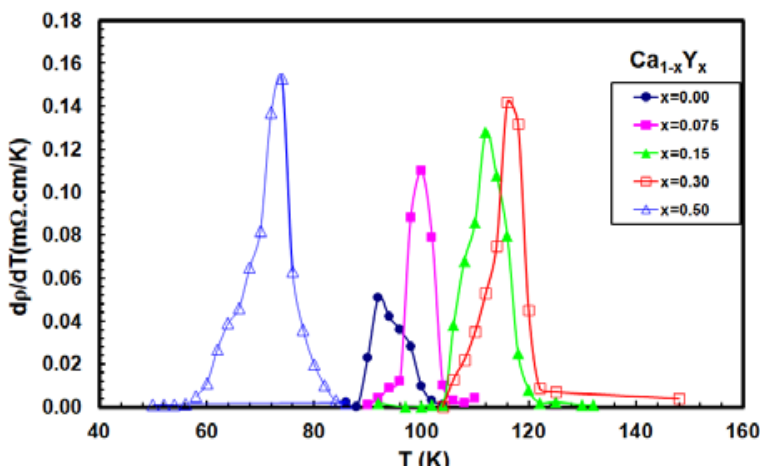

Figure 2. $\mathrm{d} \rho / \mathrm{dT}$ versus temperature for $\mathrm{Bi}_{2} \mathrm{Sr}_{2} \mathrm{Ca}_{1-\mathrm{x}}$ $\mathrm{Y}_{\mathrm{x}} \mathrm{Cu}_{2} \mathrm{O}_{\mathrm{y}}$ samples.

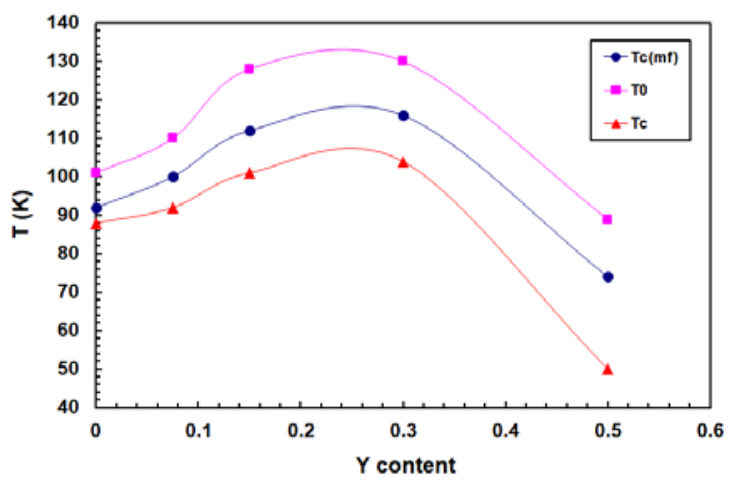

(a)

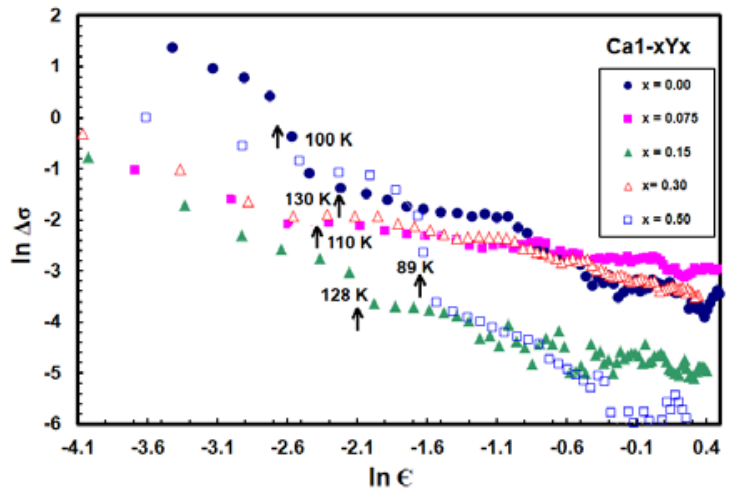

Figure 3. Ln $\Delta \sigma$ against $\mathrm{Ln} C$ for $\mathrm{Bi}_{2} \mathrm{Sr}_{2} \mathrm{Ca}_{1-\mathrm{x}}$ $\mathrm{Y}_{\mathrm{x}} \mathrm{Cu}_{2} \mathrm{O}_{\mathrm{y}}$ samples.

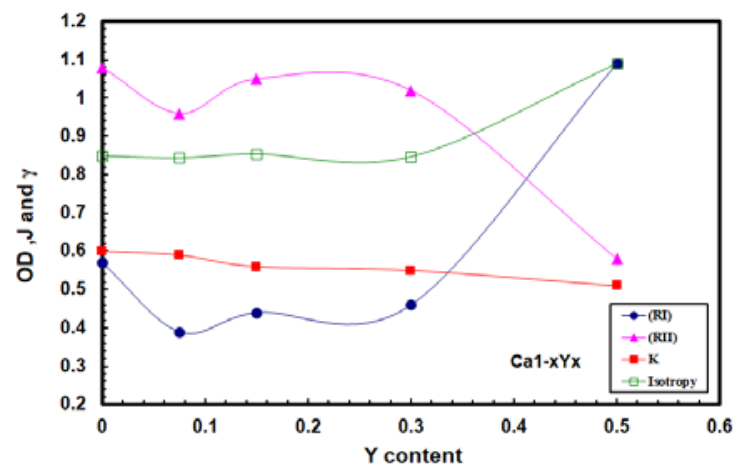

(b)

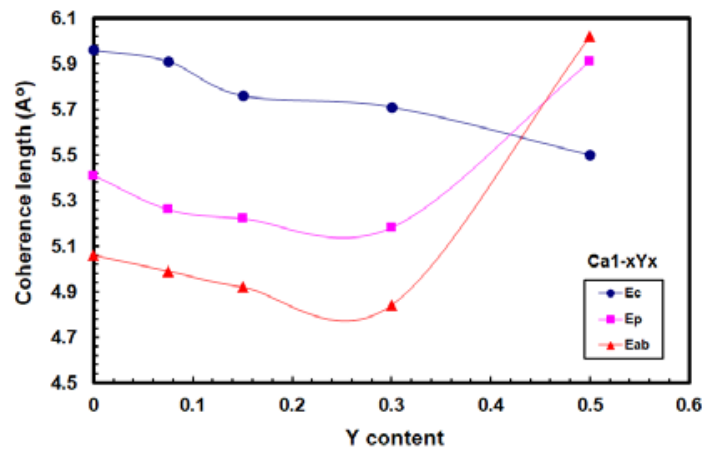

(c)

Figure 4. (a): $\mathrm{T}_{\mathrm{c}}, \mathrm{T}_{\mathrm{c}}$ mf and $\mathrm{T}_{\mathrm{o}}$ versus $\mathrm{Y}$ for $\mathrm{Bi}_{2} \mathrm{Sr}_{2} \mathrm{Ca}_{1-\mathrm{x}} \mathrm{Y}_{\mathrm{x}} \mathrm{Cu}_{2} \mathrm{O}_{\mathrm{y}}$ samples; (b): Order parameter, Interlayer coupling and anisotropy versus $\mathrm{Y}$ for $\mathrm{Bi}_{2} \mathrm{Sr}_{2} \mathrm{Ca}_{1-\mathrm{x}} \mathrm{Y}_{\mathrm{x}} \mathrm{Cu}_{2} \mathrm{O}_{\mathrm{y}}$ samples; (c): Coherence lengths versus $\mathrm{Y}$ for $\mathrm{Bi}_{2} \mathrm{Sr}_{2} \mathrm{Ca}_{1-\mathrm{x}}$ $\mathrm{Y}_{\mathrm{x}} \mathrm{Cu}_{2} \mathrm{O}_{\mathrm{y}}$ samples.

Anyhow, more than one region has been reported in pure and doped Y: 123 samples [27, 28, 32]. While, most of pure BSCCO systems are 2D behavior in the critical field region. Normally, the crossover occurs either from $3 \mathrm{D}$ to $2 \mathrm{D}$ or from 2D to $1 \mathrm{D}$ in BSCCO systems due to the effect of radiation [20,34-36]. But in the present case, 
the crossover is generally observed from 3D to 2D. Actually, the critical field region is controlled by the critical fluctuation results from the small mean free path of the charge carriers and also the short coherence length produced as the carrier concentration is changed [34-37]. This is, of course, due to the formation of high $\mathrm{T}_{\mathrm{c}}$ phase produced by $\mathrm{Y}$ up to $\mathrm{x}=0.30$ as reported for $\mathrm{T}_{\mathrm{c}}$ variation. It is also reported that the carrier concentration / $\mathrm{Cu}$ ions is decreased by $\mathrm{x}$ up to 0.30 , followed by a decrease at $\mathrm{x}=0.50$ [24], in consistent with the present behavior.

On the other hand, the interlayer coupling strength $k$ is also calculated by using equation (7), and their values are used for calculating c-axis coherence length at $0 \mathrm{~K} \quad \xi_{\mathrm{c}}(0)$, in which $\mathrm{d}=\mathrm{c} / 2=$ for $\mathrm{Bi}$ : 2212 systems [38]. For polycrystalline samples, $\xi_{\mathrm{p}}(0)$ given by [29]:

$$
\frac{1}{\xi_{p}(0)}=\frac{1}{4}\left[\frac{1}{\xi_{c}(0)}+\left(\frac{1}{\xi_{c}^{2}(0)}+\frac{8}{\xi_{a b}^{2}(0)}\right)^{\frac{1}{2}}\right]
$$

$\xi_{\mathrm{ab}}(0)$ is calculated in terms of $\xi_{\mathrm{p}}(0)$ and $\xi_{c}(0)$ values. Then, anisotropy parameter $\gamma=\xi_{a b}(0) / \xi_{c}(0)$, is easily obtained. However, the variation of $k$ and $\gamma$ against $\mathrm{x}$ is shown in Figure 4 (b), and similar values are listed in Tables 1 and 2 . The values of $\gamma$ are nearly constant by increasing Y content up to 0.30 , followed by a sharp increase at $\mathrm{x}=0.50 . \gamma$ is increased from 0.85 for $\mathrm{x}=0.30$ up to 1.09 for $\mathrm{x}=0.50$. But $k$ is gradually decreased by increasing $\mathrm{x}$ up to 0.50 . Figure 4 (c) shows the behaviors of $\xi_{\mathrm{c}}(0), \xi_{\mathrm{ab}}(0)$ and $\xi_{\mathrm{p}}(0)$ parameters against Y content and similar values are listed in Tables 2 . It is clear that $\xi_{\mathrm{c}}(0)$ is gradually decreased by $\mathrm{x}$ up to 0.50 , which is consistent with the behaviors of c-axis and $k$. While $\xi_{\mathrm{ab}}(0)$ and $\xi_{\mathrm{p}}(0)$ are decreased by $\mathrm{x}$ up to 0.30 , followed by an increase at $\mathrm{x}$ $=0.50$, which is consistent with the behaviors of $T_{c}$, carrier density and order parameter. Actually, the Bi: 2212 system is essentially 2D with two $\mathrm{Cu}-\mathrm{O}_{2}$ planes which are manifest in the lower values of $k$ and higher degree of anisotropy as compared to Y: 123 systems [38]. However, the decrease of $k$ against $\mathrm{Y}$ content suggested that the system has higher anisotropy, and consequently the carrier density should be decreased, as reported [24]. It has been also reported that the doping up to considerable level produces depletion for the excess of oxygen, thereby improving the metalcity of Bi-O layer [39], which is also observed in the resistivity curves for the present samples.

The upper critical fields along the c-axis and a-b plane, and critical current density at $0 \mathrm{~K} \mathrm{~J}(0 \mathrm{~K})$ are estimated by the following relations [3, 40, 41]:

$$
\begin{aligned}
& B_{I_{I}}(a b)=\frac{\varphi_{0}}{2 \pi \xi_{c}(0) \xi_{a b}(0)}, B_{I_{I}}(c)=\frac{\varphi_{0}}{2 \pi \xi_{a b}^{2}(0)} \\
& J_{c}(0)=\frac{2 \varphi_{0}}{\sqrt{6} \pi \lambda^{2}(0) \xi_{p}(0)}
\end{aligned}
$$

where $\varphi_{0}$ is quantum flux given by $\varphi_{0}=h / 2 e=2.07 \times 10^{-15}\left(\mathrm{web} / \mathrm{m}^{2}\right)$, and $\lambda$ is London penetration depth at $0 \mathrm{~K}$ which is about $250 \mathrm{~nm}$ for Bi:2212 superconductors [42]. However, the behaviors of $\mathrm{B}$ and $\mathrm{J}(0 \mathrm{~K})$ against $\mathrm{Y}$ content are shown in Figures 5(a) and 5 (b). Similar values are listed in Table 2. It is clear that $\mathrm{B}_{\mathrm{ab}}, \mathrm{B}_{\mathrm{c}}$ and $\mathrm{J}(0$ $\mathrm{K}$ ) are increased by $\mathrm{Y}$ content up to 0.30 , followed by a decrease for $\mathrm{Y}=0.50$. This is due to the enhancement of flux pinning, which may be ascribed by increasing the pinning centers in these types of samples [42, 43].

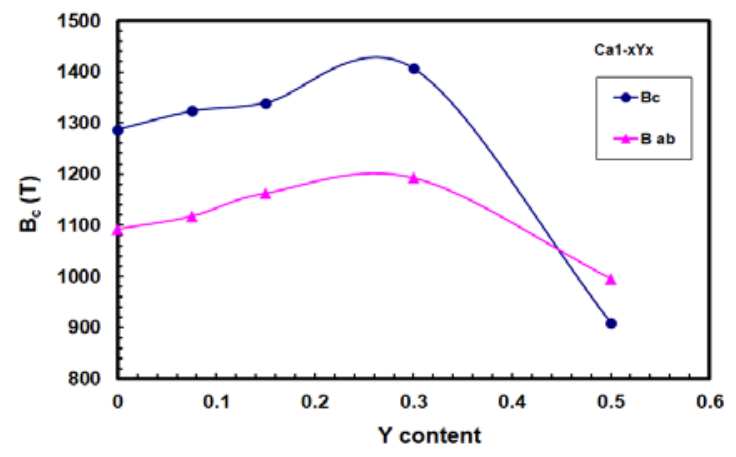

(a)

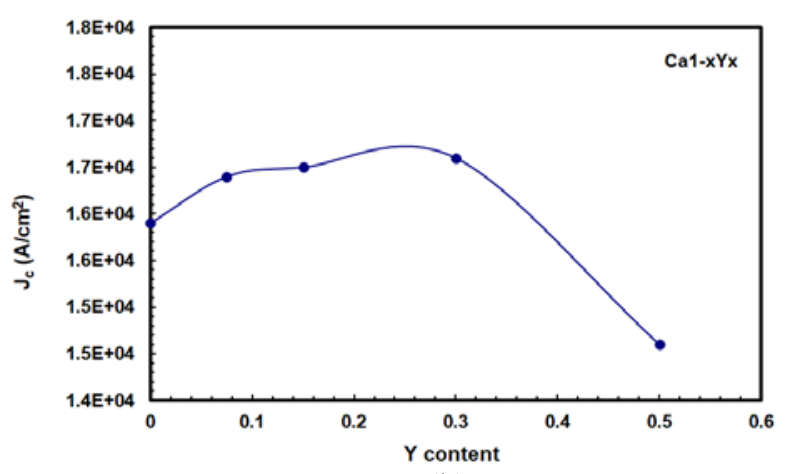

(b)

Figure 5. (a): Critical magnetic fields versus $\mathrm{Y}$ for $\mathrm{Bi}_{2} \mathrm{Sr}_{2} \mathrm{Ca}_{1-\mathrm{x}} \mathrm{Y}_{\mathrm{x}} \mathrm{Cu}_{2} \mathrm{O}_{\mathrm{y}}$ samples; (b): Critical current density versus $\mathrm{Y}$ for $\mathrm{Bi}_{2} \mathrm{Sr}_{2} \mathrm{Ca}_{1-\mathrm{x}} \mathrm{Y}_{\mathrm{x}} \mathrm{Cu}_{2} \mathrm{O}_{\mathrm{y}}$ samples.

Anyhow, a universal dome- shaped $\mathrm{T}_{\mathrm{c}}$ versus carrier concentration has been observed in Bi: 2212 system [43]. It is has been also reported that $T_{c}$ increases with increasing carrier concentration until it passes through a maximum, after which it decreases and becomes zero above an optimum value of concentration [44]. Therefore, 
the reason for the enhancement in $\mathrm{B}$ and $\mathrm{J}_{\mathrm{c}}$ up to $\mathrm{Y}=0.30$ is due to the change of carrier concentration brought by the replacement of $\mathrm{Ca}^{2+}$ by $\mathrm{Y}^{3+}$ ions in $\mathrm{Bi}: 2212$ system. This leads to electronic or chemical inhomogeneity in the charge reservoir layer ( $\mathrm{BiO} / \mathrm{SrO})$, and supplies the charge carriers to the $\mathrm{CuO}_{2}$ planes through which the actual super-current is believed to flow [45-47]. So, addition of Y decreased the hole concentration and the system goes to optimally doped condition and leading to the enhancement of the above physical parameters. The reduction of these parameters again at $\mathrm{Y}=0.50$ is due to enhancement of anisotropy along with the decrease of coupling between the $\mathrm{CuO}_{2}$ planes. This is of course will helps for producing weak links inhibiting the flow of supercurrent, and reduces the values of $\mathrm{B}$ and $\mathrm{J}$. This is also consistent with the values of OD for the $\mathrm{Y}=0.50$. Similar behavior is reported for the behavior of critical currents of $\mathrm{Bi}: 2212$ doped by Er, Fe and $\mathrm{Ni}$ at $\mathrm{Cu}$ site [48]. This is explained by increasing the pinning force density through the weak link and shifts the magnetic irreversibility line towards higher field values.

Table 1. $\mathrm{T}_{\mathrm{c}}, T_{c}^{\mathrm{mf}}, \mathrm{T}_{0}, \mathrm{~J}, \lambda_{\mathrm{RI}}$ and $\lambda_{\mathrm{RII}}$ for $\mathrm{Bi}_{2} \mathrm{Sr}_{2} \mathrm{Ca}_{1-\mathrm{x}} \mathrm{Y}_{\mathrm{x}} \mathrm{Cu}_{2} \mathrm{O}_{\mathrm{y}}$ samples.

\begin{tabular}{lllllll}
\hline $\mathbf{x}$ & $\begin{array}{l}\mathbf{T}_{\mathbf{c}} \\
(\mathbf{K})\end{array}$ & $\begin{array}{l}T_{c}^{\mathrm{mf}} \\
(\mathbf{K})\end{array}$ & $\begin{array}{l}\mathbf{T}_{\mathbf{o}} \\
(\mathbf{K})\end{array}$ & $\boldsymbol{K}$ & $\boldsymbol{\lambda}_{\text {RI }}$ & $\boldsymbol{\lambda}_{\text {RII }}$ \\
\hline 0.00 & 88 & 92 & 101 & 0.60 & $0.57(3 \mathrm{D})$ & $1.08(2 \mathrm{D})$ \\
0.075 & 92 & 100 & 110 & 0.59 & $0.39(3 \mathrm{D})$ & $0.96(2 \mathrm{D})$ \\
0.15 & 101 & 112 & 128 & 0.56 & $0.44(3 \mathrm{D})$ & $1.05(2 \mathrm{D})$ \\
0.30 & 104 & 116 & 130 & 0.55 & $0.46(3 \mathrm{D})$ & $1.02(2 \mathrm{D})$ \\
0.50 & 50 & 74 & 89 & 0.51 & $1.10(2 \mathrm{D})$ & $0.58(3 \mathrm{D})$ \\
\hline
\end{tabular}

Table 2. $\xi_{\mathrm{c}}(0), \xi_{\mathrm{p}}, \xi_{\mathrm{ab}}, \mathrm{B}_{\mathrm{c}}, \mathrm{B}_{\mathrm{ab}}$ and for $\mathrm{Bi}_{2} \mathrm{Sr}_{2} \mathrm{Ca}_{1-\mathrm{x}} \mathrm{Y}_{\mathrm{x}} \mathrm{Cu}_{2} \mathrm{O}_{\mathrm{y}}$ samples.

\begin{tabular}{llllllll}
\hline $\mathbf{X}$ & $\begin{array}{l}\xi_{\mathbf{c}}(\mathbf{0}) \\
(\AA)\end{array}$ & $\begin{array}{l}\xi_{\mathrm{p}} \mathbf{( 0 )} \\
\mathbf{( \mathbf { A } )}\end{array}$ & $\begin{array}{c}\xi_{\mathrm{ab}}(\mathbf{0}) \\
\mathbf{( \AA )}\end{array}$ & $\gamma$ & $\begin{array}{l}\mathbf{B}_{\mathbf{c}}(\mathbf{0}) \\
\mathbf{( T )}\end{array}$ & $\begin{array}{l}\mathbf{B}_{\mathbf{a b}}(\mathbf{0}) \\
\mathbf{( T )}\end{array}$ & $\begin{array}{c}\mathbf{J}(\mathbf{0}) \\
\left(\mathbf{A} / \mathbf{c m}^{2}\right)\end{array}$ \\
\hline 0.00 & 5.96 & 5.41 & 5.66 & 0.85 & 1287.4 & 1093 & $1.59 \mathrm{E}+04$ \\
0.075 & 5.91 & 5.26 & 4.99 & 0.84 & 1323.8 & 1118 & $1.64 \mathrm{E}+04$ \\
0.15 & 5.76 & 5.22 & 4.92 & 0.85 & 1339.8 & 1163 & $1.65 \mathrm{E}+04$ \\
0.30 & 5.71 & 5.18 & 4.84 & 0.85 & 1407.1 & 1193 & $1.66 \mathrm{E}+04$ \\
0.50 & 5.50 & 5.91 & 6.02 & 1.09 & 909.5 & 996 & $1.46 \mathrm{E}+04$ \\
\hline
\end{tabular}

The IR spectra of Bi:2212 superconductors reflect the contributions of electronic response of the charge carriers and lattice vibrations. Previous work of IR based on Bi:2201, Bi:2212 and Bi:2223 shows nine IR different active modes at $95 \mathrm{~cm}^{-1}, 157,200,252,298,345,460,566,632 \mathrm{~cm}^{-1}[49,50]$. While some other reports indicate that these modes are shifted to higher wave number $(600-750) \mathrm{cm}^{-1}$ by changing either oxygen deficient or carrier concentration through doping method [51]. However, the IR spectra shown in Figure 6 show two different modes of absorption above $700 \mathrm{~cm}^{-1}$ as indicated in Table 3 . The first at 716, 714, 714, 714 and $716 \mathrm{~cm}^{-1}$, for the samples, respectively; and the second at 732, 728, 730, 732 and $730 \mathrm{~cm}^{-1}$. On the other hand, The FTIR spectra shown in Figure 7 show unique mode of transmission above close to $600 \mathrm{~cm}^{-1}$ as indicated in Table 3 . It is obtained at 599 , 595, 590, 594 and $603 \mathrm{~cm}^{-1}$ for the samples, respectively.

The nature of dopants in BSCCO is considerably simplified by involving $\mathrm{O}_{\delta}$. These dopants must be somewhere in the lattice, but they could not identified in the ultrahigh resolution STM studies [52]. A model of $\mathrm{O}_{\delta}$ dopant makes several predictions that can be easily tested against the observed IR spectra [22]. In a marginally stable elastic network [2], equilibrium conditions require approximate equality of local atomic forces. The highest frequency $\omega_{\mathrm{D}}$ of an $\mathrm{O}-\mathrm{O}$ defect pair scales with its reduced mass $\mu_{\mathrm{D}}$ against $\mu_{\mathrm{H}}$, the reduced mass of the host $\mathrm{Cu}-$ O LO mode, $\omega_{\mathrm{H}}$. Thus $\mu_{\mathrm{D}} \omega_{\mathrm{D}}{ }^{2}=\mu_{\mathrm{H}} \omega_{\mathrm{H}}{ }^{2}$ and with $\mathrm{M}(\mathrm{Cu})=4 \mathrm{M}(\mathrm{O}), \omega_{\mathrm{D}}=1.26 \omega_{\mathrm{H}}$. The maximum LO neutron peak energy is $\sim 75 \mathrm{meV}=600 \mathrm{~cm}^{-1}$ in $\mathrm{Bi}_{2} \mathrm{Sr}_{2} \mathrm{CaCu}_{2} \mathrm{O}_{8+\delta}$, so the maximum frequency for an LO defect mode based on $\mathrm{O}-\mathrm{O}$ pairs is $\sim 750 \mathrm{~cm}^{-1}$. The maximum value obtained in the extended Drude analysis of the infrared spectra at optimal doping is $750 \mathrm{~cm}^{-1}$, [51] in agreement with the present data $\left(730 \mathrm{~cm}^{-1}\right)$.

However, in our recent work based on the same samples, we have shown that both effective Cu valance and carrier concentration/ $\mathrm{Cu}$ ion are decreased by increasing $\mathrm{x}$ up to 0.30 , followed by a decrease with further increase of $x$ up to 0.50 [24]. The vice is versa for critical fields and currents. Furthermore, the order parameter exponents are shifted from 3D to 2D and these effects are nearly shifted the IR spectra to a little bit lower values, in agreement with the reported for critical temperature, hardness, oxygen deficient, $\mathrm{Cu}$ valance and carrier concentration/ $\mathrm{Cu}$ ions. However, substitution of $\mathrm{Y}$ above 0.30 in Bi:2212 verifies the following points: (i) shifting the order parameter from 3D to $2 \mathrm{D}$ in $\mathrm{R}_{\mathrm{I}}$, and from $2 \mathrm{D}$ to quesi $2 \mathrm{D}$ in $\mathrm{R}_{\mathrm{II}}$; decreasing the critical fields and current; shifting the IR spectra to lower/higher values. The consistency of these points gives a fair degree of certainty to the suggestion of Y substitution in Bi: 2212 system. 


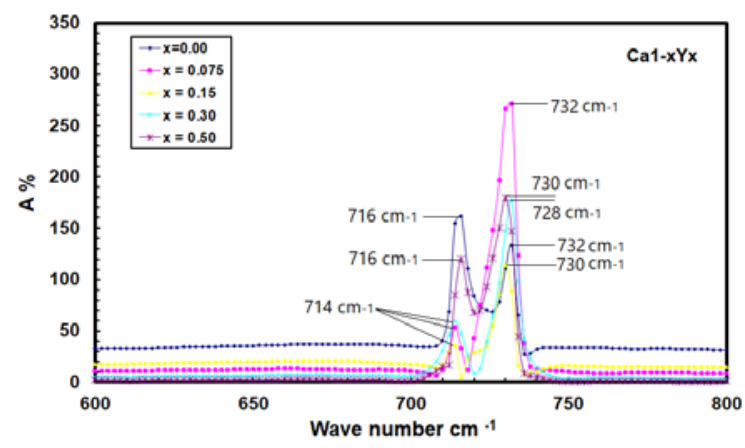

Figure 6. IR spectra for $\mathrm{Bi}_{2} \mathrm{Sr}_{2} \mathrm{Ca}_{1-\mathrm{x}} \mathrm{Y}_{\mathrm{x}} \mathrm{Cu}_{2} \mathrm{O}_{\mathrm{y}}$ samples

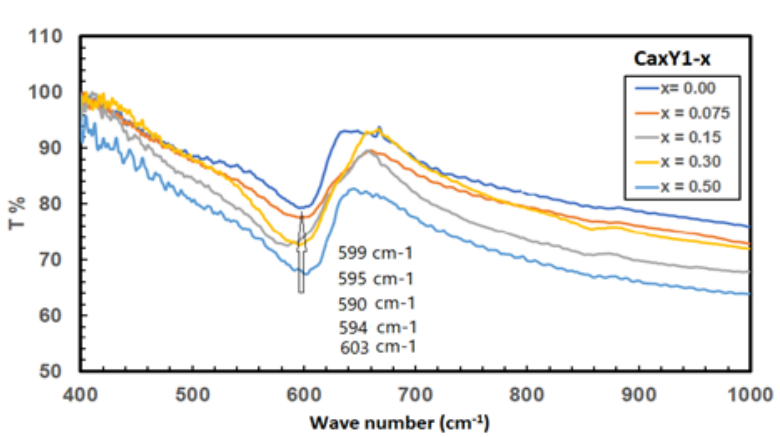

Figure 7. FTIR spectra for $\mathrm{Bi}_{2} \mathrm{Sr}_{2} \mathrm{Ca}_{1-\mathrm{x}} \mathrm{Y}_{\mathrm{x}} \mathrm{Cu}_{2} \mathrm{O}_{\mathrm{y}}$ samples.

Table 3. IR spectra of $\mathrm{Bi}_{2} \mathrm{Sr}_{2} \mathrm{Ca}_{1-\mathrm{x}} \mathrm{Y}_{\mathrm{x}} \mathrm{Cu}_{2} \mathrm{O}_{\mathrm{y}}$ samples

\begin{tabular}{llll}
\hline $\mathbf{x}$ & $\begin{array}{l}\mathbf{A}_{\mathbf{1}} \\
\left(\mathbf{c m}^{-\mathbf{1}}\right)\end{array}$ & $\begin{array}{l}\mathbf{A} \mathbf{2} \\
\left(\mathbf{c m}^{-\mathbf{1}}\right)\end{array}$ & $\begin{array}{l}\mathbf{T} \\
\left(\mathbf{c m}^{-\mathbf{1}}\right)\end{array}$ \\
\hline 0.00 & 716 & 732 & 599 \\
0.075 & 714 & 728 & 595 \\
0.15 & 714 & 730 & 590 \\
0.30 & 714 & 732 & 594 \\
0.50 & 716 & 730 & 603 \\
\hline
\end{tabular}

\section{Conclusions}

Excess conductivity and IR spectra of $\mathrm{Bi}_{2} \mathrm{Sr}_{2} \mathrm{Ca}_{1-x} \mathrm{Y}_{\mathrm{x}} \mathrm{Cu}_{2} \mathrm{Oy}$ superconductors are investigated. We have shown a crossover from $3 \mathrm{D}$ to $2 \mathrm{D}$ with $\mathrm{x} \leq 0.30$, and from $2 \mathrm{D}$ to quasi $2 \mathrm{D}$ for $\mathrm{x}=0.50$. The substitution of $\mathrm{Y}$ up to 0.30 improved the critical magnetic fields and current followed by a decrease at $\mathrm{x}=0.50$. Similar behavior could be obtained for the three different modes recorded in the range of (714-732) $\mathrm{cm}^{-1}$. A good correlation between fluctuation study and IR spectra gives a fair degree of certainty to the suggestion of Y substitution in Bi: 2212 system.

\section{References}

[1] Gotō T, Niimi A. Magnetic Properties of Superconducting Pb-Bi System Alloy Filaments Produced by GlassCoated Melt Spinning. Japanese Journal of Applied Physics. 1988;27(2R):209.

[2] Talantsev EF, Strickland NM, Hoefakker P, Xia JA, Long NJ. Critical current anisotropy for second generation HTS wires. Current Applied Physics. 2008;8(3-4):388-390.

[3] Khan NA, Hassan N, Nawaz S, Shabbir B, Khan S, Rizvi AA. Effect of Sn substitution on the paraconductivity of polycrystalline $\mathrm{Cu} 0.5 \mathrm{~T} 10.5 \mathrm{Ba} 2 \mathrm{Ca} 2 \mathrm{Cu} 3-\mathrm{ySnyO} 10-\delta$ superconductors. Journal of Applied Physics. 2010;107(8):083910.

[4] Giannini E, Gladyshevskii R, Clayton N, Musolino N, Garnier V, Piriou A, Flükiger R. Growth, structure and physical properties of single crystals of pure and Pb-doped Bi-based high Tc superconductors. Current Applied Physics. 2008;8(2):115-119.

[5] Haraguchi T, Takayama S, Kiuchi M, Otabe ES, Matsushita T, Yasuda T, Okayasu S, Uchida S, Shimoyama J, Kishio K. Influence of anisotropy and pinning centers on critical current properties in Bi-2212 superconductors. Physica C: Superconductivity and Its Applications. 2006;445:123-127.

[6] Aliev FG. Generation of DC electric fields due to vortex rectification in superconducting films. Physica C: Superconductivity and Its Applications. 2006;437:1-6.

[7] Bahrs S, Bruchhausen A, Goñi AR, Nieva G, Fainstein A, Fleischer K, Richter W, Thomsen C. Effect of light on the reflectance anisotropy and chain-oxygen related Raman signal in untwinned, underdoped crystals of YBa2Cu3O7- $\delta$. Journal of Physics and Chemistry of Solids. 2006;67(1-3):340-343.

[8] Ghosh AK, Bandyopadhyay SK, Barat P, Sen P, Basu AN. Excess-conductivity analysis of $\alpha$ irradiated polycrystalline Bi-2212 superconductor. Physica C: Superconductivity. 1995;255(3-4):319-323.

[9] Ghosh AK, Basu AN. Dimensional exponents of Gaussian and critical fluctuations in Bi 2 Sr 2 CaCu 2 O 8+delta processed under different conditions. Supercond. Sci. Technol. 2000;13:343. 
[10] Ghosh AK, Bandyopadhyay SK, Basu AN. Dimensional Crossover Temperature in Presence of Two Different Josephson Couplings in High T c Superconductors. Modern Physics Letters B. 1997;11(23):10131020.

[11] Fisher DS, Fisher MP, Huse DA. Thermal fluctuations, quenched disorder, phase transitions, and transport in type-II superconductors. Physical Review B. 1991;43(1):130-159.

[12] Vidal F, Viera JA, Maza J, Ponte JJ, Garcia-Alvarado F, Moran E, Amador J, Cascales C, Castro A, Casais MT, Rasines I. Excess electrical conductivity in polycrystalline Bi-Ca-Sr-Cu-O compounds and thermodynamic fluctuations of the amplitude of the superconducting order parameter. Physica C: Superconductivity. 1988;156(5):807-816.

[13] Mumtaz M, Hasnain SM, Khurram AA, Khan NA. Fluctuation induced conductivity in $(\mathrm{Cu} 0.5 \mathrm{~T} 10.5-\mathrm{xKx})$ Ba2Ca3Cu4O12- $\delta$ superconductor. Journal of Applied Physics. 2011;109(2):023906.

[14] Esmaeili A, Sedghi H, Amniat-Talab M, Talebian M. Fluctuation-induced conductivity and dimensionality in the new Y-based Y3Ba5Cu8O18-x superconductor. The European Physical Journal B. 2011;79(4):443447.

[15] Semba K, Matsuda A, Ishii T. Normal and superconductive properties of Zn-substituted single-crystal YBa2(Cu1-xZnx)3O7- $\delta$. Physical Review B. 1994;49(14):10043-10046.

[16] Mandal P, Poddar A, Das AN, Ghosh B, Choudhury P. Excess conductivity and thermally activated dissipation studies in Bi2Sr2Ca1Cu2Ox single crystals. Physica C: Superconductivity. 1990;169(1-2):43-49.

[17] Hayashi Y, Yosida T, Kontani N, Fukui M, Sako S, Fujita T, Sasakura H, Minamigawa S, Nakahigashi K. Absence and existence of ESR in high-Tc superconductors. Physica B: Condensed Matter. 1990;165:13171318.

[18] Mun MO, Lee SI, Salk SH, Shin HJ, Joo MK. Conductivity fluctuations in a single crystal of Bi2Sr2CaCu2Ox. Physical Review B. 1993;48(9):6703-6706.

[19] Poddar A, Mandal P, Das AN, Ghosh B, Choudhury P. Electrical resistivity, magnetoresistance, magnetisation, hall coefficient and excess conductivity in Pb-doped Bi-Sr-Ca-Cu oxides. Physica C: Superconductivity. 1989;161(5-6):567-573.

[20] Mori N, Wilson JA, Ozaki H. Fluctuation conductivity in the 110-K phase of Ni-doped (Bi, Pb)-Sr-Ca-Cu-O superconductors. Physical Review B. 1992;45(18):10633-10638.

[21] Yanru R, Hanpeng L, Mingzhu L, Qingyun T, Lihua S, Zhenjin L, Xianren M, Zhenxing L. Infrared spectra of the high-Tc TlBaCaCuO superconductors. Physica C: Superconductivity. 1988;156(5):799-803.

[22] Hwang J, Timusk T, Gu GD. High-transition-temperature superconductivity in the absence of the magneticresonance mode. Nature. 2004;427(6976):714-717.

[23] Norman M. Superconductivity: Shine a light. Nature. 2004;427(6976):692.

[24] Sedky A, Al-Battat W. Effect of Y substitution at Ca site on structural and superconducting properties of Bi: 2212 superconductor. Physica B: Condensed Matter. 2013;410:227-232.

[25] Das A, Suryanarayanan R. Remarkable Influence of Heat Treatment on the Structural and Superconducting Properties of Y1-xPrxSrBaCu3O6+z. J. Phys. I. 1995;5: 623-630.

[26] Anderson PW, Zou Z. "Normal" Tunneling and" Normal" Transport: Diagnostics for the Resonating-ValenceBond State. Physical Review Letters. 1988;60(2):132-135.

[27] Aslamasov LG, Larkin AI. The influence of fluctuation pairing of electrons on the conductivity of normal metal. Physics Letters A. 1968;26(6):238-239.

[28] Lawrence WE, Doniach S.: low tempertature. In: 12th International Conference on Low Temperature Physics. Kyoto, Japan: Academic Press;1970. p. 361.

[29] Ghosh AK, Bandyopadhyay SK, Basu AN. Generalization of fluctuation induced conductivity in polycrystalline $\mathrm{Y} 1-\mathrm{xCaxBa} 2 \mathrm{Cu} 3 \mathrm{Oy}$ and $\mathrm{Bi} 2 \mathrm{Sr} 2 \mathrm{Ca} 1 \mathrm{Cu} 2 \mathrm{O} 8+\delta$ superconductors. Journal of Applied Physics. 1999;86(6):3247-3252.

[30] Ghosh AK, Bandyopadhyay SK, Barat P, Sen P, Basu AN. Fluctuation-induced conductivity of polycrystalline Y1-xCaxBa2Cu3O7- $\delta$ superconductors. Physica C: Superconductivity. 1996;264(3-4):255260.

[31] Sedky A. Fluctuation-Induced Excess Conductivity in R1-x Cax: 123 Superconductors. Journal of Low Temperature Physics. 2007;148(1-2):53-64.

[32] Ramallo MV, Torrón C, Vidal F. Fluctuation-induced diamagnetism in biperiodic layered superconductors in the weak magnetic field regime. Physica C: Superconductivity. 1994;230(1-2):97-109.

[33] Baraduc C, Buzdin A. Fluctuations in layered superconductors: different inter-plane coupling and effect on the London penetration depth. Physics Letters A. 1992;171(5-6):408-414.

[34] Ravi S, Bai VS. Excess conductivity studies in pure and Ag doped $85 \mathrm{~K}$ phase in BiSrCaCuO system. Solid State Communications. 1992;83(2):117-121.

[35] Weaver BD, Jackson EM, Summers GP, Burke EA. Atomic disorder and the transition temperature of cuprate superconductors. Physical Review B. 1992;46(2):1134-1137. 
[36] Veira JA, Maza J, Vidal F. Excess electrical conductivity in polycrystalline Y1Ba2Cu3O7- $\delta$ compounds: Beyond the mean-field region. Physics Letters A. 1988;131(4-5):310-314.

[37] Vidal F, Veira JA, Maza J, Garcia-Alvarado F, Moran E, Alario MA. Excess electrical conductivity above Tc in high-temperature superconductors, and thermal fluctuations. Journal of Physics C: Solid State Physics. 1988;21(16):L599.

[38] Mandal P, Poddar A, Das S. Excess conductivity analysis of the Bi2Sr2Ca1-xYxCu2O8+ y system: an estimation of interlayer coupling strength. Journal of Physics: Condensed Matter. 1994;6(29):5689.

[39] Samanta SB, Dutta PK, Awana VP, Gmelin E, Narlikar AV. High resolution STM/STS studies of Bi-O redox layers and superconductivity in pure and substituted Bi-2122 high-Tc cuprate. Physica C: Superconductivity. 1991;178(1-3):171-181.

[40] Ghorbani SR, Homaei M. Excess fluctuation Conductivity and Superconducting parameters of CaLa-doped Nd-123. Modern Physics Letters B. 2011;25(23):1915-1924.

[41] Petrović A, Fasano Y, Lortz R, Decroux M, Potel M, Chevrel R, Fischer Ø. Unconventional resistive transitions in the extreme type-II superconductor Tl2Mo6Se6. Physica C: Superconductivity and Its Applications. 2007;460:702-703.

[42] Sedky A. On the study of Müllers model in high-temperature superconductors. Journal of Magnetism and Magnetic Materials. 2004;277(3):293-297.

[43] Mandal P, Poddar A, Ghosh B, Choudhury P. Variation of Tc and transport properties with carrier concentration in Y- and Pb-doped Bi-based superconductors. Phys. Rev. B. 1991;43:13102-13111.

[44] Matsuda A, Kinoshita K, Ishii T, Shibata H, Watanabe T, Yamada T. Electronic properties of Ba2Y1-xPrxCu3O7- $\delta$. Physical Review B. 1988;38(4):2910-2913.

[45] Aloysius RP, Guruswamy P, Syamaprasad U. Highly enhanced critical current density in Pr-added (Bi, Pb)2212 superconductor. Superconductor Science and Technology. 2005;18(5):L23.

[46] Biju A, Sarun PM, Aloysius RP, Syamaprasad U. Structural and superconducting properties of neodymium added (Bi, Pb) 2Sr2CaCu2Oy. Materials Research Bulletin. 2007;42(12):2057-2066.

[47] Biju A, Vinod K, Aloysius RP, Syamaprasad U. Improved superconducting properties by La addition in (Bi, $\mathrm{Pb}$ )-2212 bulk superconductor. Journal of Alloys and Compounds. 2007;431(1-2):49-55.

[48] Ilonca G, Yang TR, Pop AV, Stiufiuc G, Stiufiuc R, Lung C. Critical currents of Bi: 2212 doped by Fe and Ni. Physica C: Superconductivity. 2003;388:425-426.

[49] Hudáková N, Knížek K, Hejtmánek J. The infrared properties of Bi2Y0.1Sr1.9CaCu2O8+ $\delta$ superconductor. Physica C: Superconductivity. 2004;406(1-2):58-62.

[50] Xu G, Pu Q, Liu B, Zhang J, Zhang C, Ding Z, Zhang Y. Different Tc-suppression rates between Mn doped La214 and Bi2201 systems. Physica C: Superconductivity. 2003;390(1):75-79.

[51] Phillips JC. Superconductive excitations and the infrared vibronic spectra of BSCCO. Physica Status Solidi (B). 2005;242(1):51-57.

[52] Lang KM, Madhavan V, Hoffman JE, Hudson EW, Eisaki H, Uchida S, Davis JC. Imaging the granular structure of high-Tc superconductivity in underdoped $\mathrm{Bi}_{2} \mathrm{Sr}_{2} \mathrm{CaCu}_{2} \mathrm{O}_{+\delta}$. Nature. 2002;415:412-416.

(C) 2019 by the author(s). This work is licensed under a Creative Commons Attribution 4.0 International License (http://creativecommons.org/licenses/by/4.0/). Authors retain copyright of their work, with first publication rights granted to Tech Reviews Ltd. 